\title{
Binding of heparin to human thyroglobulin (Tg) involves multiple binding sites including a region corresponding to a binding site of rat $\mathrm{Tg}$
}

\author{
Simonetta Lisi, Aldo Pinchera, Robert T McCluskey ${ }^{1}$, Luca Chiovato ${ }^{2}$ and Michele Marinò \\ Department of Endocrinology, University of Pisa, Via Paradisa 2, 56124 Pisa, Italy, ${ }^{1}$ Pathology Research Laboratory, Massachusetts General Hospital, \\ Harvard Medical School, 149 13th Street, Charlestown, Massachusetts 02129, USA and ${ }^{2}$ University of Pavia, Fondazione Salvatore Maugeri, IRCCS, \\ Pavia, Italy \\ (Correspondence should be addressed to Michele Marinò; Email: m.marino@endoc.med.unipi.it)
}

\begin{abstract}
Objective: Binding of thyroglobulin (Tg) to heparin allows efficient $\mathrm{Tg}$ interaction with its endocytic receptor, megalin. Rat $\mathrm{Tg}(\mathrm{rTg})$ binds to heparin using an exposed carboxyl terminal region (RELPSRRLKRPLPVK, Arg2489-Lys2503) rich in positively charged residues which is, however, not entirely conserved in human Tg (hTg) (Arg2489-Glu2503, REPPARALKRSLWVE). Here, we investigated whether and how this difference affects binding of heparin.

Design: To compare binding of heparin to rTg and hTg. To investigate the role of the sequence 24892503 using a peptide-based approach.

Methods: Binding of biotin-labeled heparin to rTg, hTg and to Tg peptides was measured in solid phase assays.

Results: Heparin bound to rTg with moderately high affinity $\left(K_{\mathrm{d}}: 34.2 \mathrm{nmol} / \mathrm{l}, K_{\mathrm{i}}: 37.6 \mathrm{nmol} / \mathrm{l}\right)$ and to hTg with lower affinity $\left(K_{\mathrm{d}}: 118 \mathrm{nmol} / \mathrm{l}, K_{\mathrm{i}}: 480 \mathrm{nmol} / \mathrm{l}\right)$ and to a lower extent. Binding was dosedependent and saturable, and was reduced by several specific competitors (Tg itself, unlabeled heparin, lactoferrin). Heparin bound to synthetic peptides corresponding to the rat (rTgP) and to the human (hTgP) Tg sequence 2489-2503. Heparin bound to rTgP to a greater extent and with greater affinity than to hTgP. An antibody against hTgP reduced binding of heparin to intact hTg by $30 \%$, suggesting that in hTg this region is, in part, involved in heparin binding, but also that other regions account for most of the binding. Starting from the sequence of rTgP, we designed 6 synthetic 'mutant' peptides by replacing one amino acid residue of $\mathrm{rTgP}$ with the corresponding residue of the sequence of hTgP. Heparin bound to 5 of 6 mutant peptides to a lower extent and with lower affinity than to rTgP.

Conclusions: In spite of a reduced binding ability of the sequence 2489-2503, hTg binds to heparin, in part, using alternative, as yet unidentified, binding sites. Substitution of both positive and neutral residues within the sequence 2489-2503 reduced heparin-binding, suggesting that not only charge, but also sequence and/or conformation, may account for the heparin-binding ability of this region of Tg.
\end{abstract}

European Journal of Endocrinology 146 591-602

\section{Introduction}

Binding of proteins to heparin and to heparin-like molecules, namely glycosaminoglycans (GAGs) occurs mainly through the interaction of negatively charged regions of GAGs with positively charged regions of proteins $(1-4)$. The majority of heparin-binding proteins bind to heparin via short sequences rich in positively charged amino acid residues (arginine and lysine), known as Cardin and Weintraub's heparin-binding consensus motifs $(1-4)$. However, certain heparinbinding proteins do not contain in their sequence any Cardin and Weintraub's motifs $(1-4)$. In these cases, binding to heparin occurs via clusters of positively charged amino acid residues that are distant in the sequence and are brought together by the folding of the protein $(1-4)$.

The heparin-binding ability of proteins allows their interaction with GAGs. GAGs, which comprise heparan sulfate proteoglycans (HSPGs), chondroitin sulfates and hyaluronic acid, are among the major components of the extracellular matrix and are attached to the surface of most vertebrate cells (2-4). GAGs are involved in several important biological processes, including cellcell and cell-matrix interactions, cell proliferation and growth. In addition, GAGs, especially HSPGs, facilitate receptor-mediated endocytosis of certain proteins that bind to cell surface HSPGs $(2-4)$. This interaction 
occurs via side-by-side binding sites for the receptor and for heparin $(2-4)$.

Recently, we have studied extensively the endocytic pathway of thyroglobulin $(\mathrm{Tg})$, the precursor of thyroid hormones (5-9). Tg is synthesized by thyroid epithelial cells (thyrocytes) and released into the lumen of thyroid follicles (10-13). Hormone secretion mainly occurs following fluid phase $\mathrm{Tg}$ uptake by thyrocytes from the colloid and proteolytic cleavage along the lysosomal pathway (10-13). In addition, Tg can be taken up by receptor-mediated endocytosis, in this case resulting in its recycling into the colloid or in its transepithelial transport (transcytosis) across thyrocytes (10-13). We found that megalin, a member of the low density lipoprotein receptor family $(14-18)$ present on the apical surface of thyrocytes $(19,20)$, is responsible for $\mathrm{Tg}$ transcytosis $(7,8,10)$, a process involved in the regulation of hormone release.

Several lines of evidence led us to the conclusion that efficient binding of rat $\mathrm{Tg}$ to megalin requires $\mathrm{Tg}$ binding to HSPGs, which occurs via functionally related binding sites for megalin and for heparin $(6,9)$. First, we found that rat $\mathrm{Tg}$ is a heparin-binding protein and that heparin inhibits its binding to megalin, both in solid phase assays (21) and in cultured thyroid cells (5). Secondly, we obtained evidence that the major heparin and megalin binding sites of rat $\mathrm{Tg}$ reside in its carboxyl terminal portion (6). Thirdly, we identified a major heparin-binding site of rat $\mathrm{Tg}$ and found that occupation of this binding site results in impaired $\mathrm{Tg}$ interactions with megalin (6). Fourthly, we obtained evidence that rat $\mathrm{Tg}$ binds to HSPGs in vitro and in cultured thyroid cells using the same heparin-binding site needed for megalin binding (9).

As mentioned above, the heparin-binding site of rat Tg which we identified is located in the carboxyl terminal portion of the recently obtained complete sequence of rat $\mathrm{Tg}$ (22). To identify this binding site, we used a 15 amino acid synthetic peptide (RELPSRRLKRPLPVK) corresponding to the rat Tg sequence Arg2489Lys2503, characterized by the presence of a Cardin and Weintraub's heparin-binding consensus motif (SRRLKRP) (6). A rabbit antibody developed against the rat $\mathrm{Tg}$ sequence Arg2489-Lys2503 precipitated native, intact $\mathrm{Tg}$, indicating that the sequence is exposed on the surface of the $\mathrm{Tg}$ molecule in its native conformation (6). In addition, this antibody markedly reduced (by 70\%) binding of heparin to $\mathrm{Tg}$, indicating that this sequence is required for heparinbinding (6).

The heparin-binding sequence we identified in rat $\mathrm{Tg}$ is entirely conserved in mouse $\mathrm{Tg}$ (23). However, by analysis of the sequence of human $\mathrm{Tg}$ (24), we found that the heparin-binding sequence of rat $\mathrm{Tg}$ is not entirely conserved (Arg2489-Glu2503, REPPARALKRSLWVE). In the present study, we investigated whether and how the different sequences of $\mathrm{Tg}$ in rodents and humans affect their heparin-binding ability. The results indicate that, in spite of a markedly reduced heparinbinding affinity of the carboxyl terminal heparin-binding site, human $\mathrm{Tg}$ does bind to heparin using, in part, alternative, as yet unidentified, heparin-binding sites. We also found that the heparin-binding affinity of the carboxyl terminal binding site of $\mathrm{Tg}$ may depend not only on charge, but also on sequence and/or conformation of the region.

\section{Materials and methods}

\section{Materials}

Human and rat Tg were prepared by tissue extraction, ammonium sulfate precipitation and column fractionation, as previously described (5-9). The source of human $\mathrm{Tg}$ was the contralateral, normal thyroid lobe of a patient subjected to total thyroidectomy for papillary thyroid carcinoma. Written consent was obtained from this patient. Rat $\mathrm{Tg}$ (rTg) was obtained from frozen rat thyroids (Pel-Freeze Biologicals, Rogers, AK, USA).

A biotin-labeled heparin-albumin complex, biotinlabeled albumin, unlabeled heparin, lactoferrin, and ovalbumin (OVA) were obtained from Sigma (St Louis, MO, USA). Alkaline phosphatase (ALP)-conjugated streptavidin was obtained from Vector (Burlingame, CA, USA).

Nine 15-mer peptides were synthesized by the Peptide-Protein Core Facility of the Massachusetts General Hospital (Charlestown, MA, USA). The sequences of these peptides and their position within the amino acid sequence of $\mathrm{Tg}$ are shown in Table 1 . The peptides were designated as reported in Table 1.

An immunoaffinity purified polyclonal rabbit antibody against one of the synthetic peptides (rTgP), raised by Cocalico (Reamston, PA, USA), was previously described and characterized (6). ALP-conjugated goat anti-human IgG was purchased from Sigma.

\section{Selection of human sera containing autoantibodies against a human Tg peptide (hTgP)}

Sera from eleven patients (one male and ten females, age range 11-67 years) with autoimmune thyroiditis seen at the Department of Endocrinology of the University of Pisa were collected and numbered from 1 to 11 . Written consent was obtained from all patients. All patients had undergone a complete clinical and laboratory thyroid evaluation, which included physical examination, thyroid ultrasonography and the following tests: free thyroid hormones (thyroxine $\left(\mathrm{FT}_{4}\right)$ and tri-iodothyronine $\left(\mathrm{FT}_{3}\right)$ RIA, Lysophase, Technogenetics SpA, Milan, Italy), thyrotropic hormone (Ultrasensitive-TSH IFMA, Delfia, Wallac, Finland), anti-Tg autoantibodies (anti-Tg MELISA, Byk Gulden SpA, Milan, Italy) and antithyroperoxidase autoantibodies (anti-TPO RIA, Sorin 
Table 1 Synthetic peptides used in heparin-binding experiments.

\begin{tabular}{llc}
\hline Peptide name & Amino acid sequence & $\begin{array}{c}\text { Amino acid position } \\
\text { in the sequence of Tg }\end{array}$ \\
\hline rTgP & RELPSRRLKRPLPVK & $\begin{array}{c}\text { Arg2489-Lys2503 } \\
\text { ArgP }\end{array}$ \\
Mutant 5 & REPPA RALKRS LWVE & - \\
Mutant 6 & REPPSRRLKRPLPVK & - \\
Mutant 7 & RELPA RRLKRPLPVK & - \\
Mutant 8 & RELPSRALKRPLPVK & - \\
Mutant 9 & RELPSRRLKRSLPVK & - \\
Mutant 10 & RELPSRRLKRPLWVK & - \\
Control peptide & RELPSRRLKRPLPVE & - \\
\hline
\end{tabular}

The residues in bold and underlined are those altered with respect to $\mathrm{rTgP}$.

Biomedica SpA, Saluggia, Italy). The diagnosis of autoimmune thyroiditis was based on the presence of hypothyroidism and circulating anti-Tg and/or antithyroperoxidase autoantibodies, with a hypoechogenic pattern of the thyroid at ultrasound examination (25). In particular, anti-Tg autoantibodies were detected at high levels in 10 of 11 patients.

The presence of serum IgGs reactive with hTgP was assessed by enzyme-linked immunoadsorbent assay (ELISA). For this purpose, 96-well microtiter plates were coated overnight at $4{ }^{\circ} \mathrm{C}$ with $\mathrm{hTgP}(1 \mathrm{mg} / \mathrm{ml})$ in phosphate buffered saline (PBS), in a $100 \mu \mathrm{l}$ volume. Wells were then blocked for $3 \mathrm{~h}$ at $4{ }^{\circ} \mathrm{C}$ with $1 \mathrm{mg} / \mathrm{ml}$ bovine serum albumin (BSA) and washed three times with PBS, $0.05 \%$ Tween-20. They were incubated for $3 \mathrm{~h}$ at room temperature with sera diluted 1:5 in PBS, $0.05 \%$ Tween-20, $0.5 \%$ BSA, either alone or in the presence of hTgP or of a control peptide (Table 1) at a $100 \mu \mathrm{g} / \mathrm{ml}$ concentration, after overnight preincubation at $4^{\circ} \mathrm{C}$. Plates were then washed and incubated with ALP-conjugated anti-human IgG (1:4000) followed by p-nitrophenyl-phosphate (Sigma). Absorbance was determined at $405 \mathrm{~nm}$.

\section{Purification and characterization of human IgGs reactive against hTgP}

Cyanogen bromide activated agarose beads (Sigma) were coupled at saturation with $\mathrm{hTgP}$ according to the manufacturer's instructions. Beads were blocked by incubation for $2 \mathrm{~h}$ at $4{ }^{\circ} \mathrm{C}$ followed by $1 \mathrm{~h}$ at room temperature with $10 \mathrm{ml}$ serum from a normal subject previously found to be devoid of anti-Tg autoantibodies. Beads were extensively washed with PBS and then incubated overnight at $4{ }^{\circ} \mathrm{C}$ with $4.5 \mathrm{ml}$ of a pool of three sera (numbers 6, 7 and 10) from patients with autoimmune thyroiditis that were found to contain autoantibodies against hTgP. Beads were extensively washed with PBS, and IgGs that had been captured by the hTgP-conjugated beads were dissociated from the beads by incubation for a few seconds with $3 \mathrm{ml}$ $0.1 \mathrm{~mol} / \mathrm{l}$ citric acid, $\mathrm{pH}$ 3.3. The beads were spun for a few seconds, the supernatant was collected and buffered by rapidly adding $600 \mu \mathrm{l}$ Tris $(2 \mathrm{~mol} / \mathrm{l}$, pH 8.0) to a final $\mathrm{pH}$ value of 7.5. The supernatant containing the anti-hTgP antibody was then dialyzed overnight at $4{ }^{\circ} \mathrm{C}$ against $2 \mathrm{l}$ PBS.

The reactivity of anti-hTgP antibody to hTgP and intact human $\mathrm{Tg}$ was tested by ELISA. For this purpose, 96-well microtiter plates were coated overnight at $4{ }^{\circ} \mathrm{C}$ with hTgP or with intact human Tg or, as controls, with a control peptide (Table 1) or with BSA, all at a concentration of $1 \mathrm{mg} / \mathrm{ml}$ in PBS. Wells were blocked with BSA as described above, washed three times with PBS, $0.05 \%$ Tween-20, and incubated for $3 \mathrm{~h}$ at room temperature with the anti-hTgP antibody diluted 1:10 in PBS, $0.05 \%$ Tween-20, 0.5\% BSA. Plates were then washed and incubated with the ALP-conjugated anti-human IgG antibody (1:4000), followed by p-nitrophenyl-phosphate. Absorbance was determined at $405 \mathrm{~nm}$.

\section{Solid phase heparin-binding assays}

Solid phase binding assays were performed as described previously $(5,6)$. Briefly, 96 -well microtiter plates were coated overnight at $4{ }^{\circ} \mathrm{C}$ with rat $\mathrm{Tg}$, human $\mathrm{Tg}$ or the synthetic peptides at various concentrations in PBS, or, as a control, with OVA. For coating, proteins were added in a volume of $100 \mu \mathrm{l} /$ well. After coating, wells were blocked with BSA, washed three times with Trisbuffered saline (TBS) containing $0.05 \%$ Tween-20 and incubated for $3 \mathrm{~h}$ at room temperature with biotinlabeled heparin $(0.1 \mu \mathrm{g} / \mathrm{ml})$ in TBS, $0.05 \%$ Tween-20, $0.5 \%$ BSA or, as a control, with biotin-labeled albumin (in which case OVA instead of BSA was used for blocking and in the binding buffer), followed by ALPconjugated streptavidin (1:3000) and p-nitrophenylphosphate (Sigma). Absorbance was determined at $405 \mathrm{~nm}$. The amount of bound heparin or bound albumin was calculated using a standard curve obtained by coating the wells with $0.1-1000 \mathrm{ng}$ biotin-labeled heparin or biotin-labeled albumin.

For inhibition experiments, biotin-labeled heparin was added to the wells alone or together with unlabeled heparin $(250 \mathrm{nmol} / \mathrm{l})$, unlabeled rat or human $\mathrm{Tg}$ 
(various concentrations), lactoferrin $(100 \mu \mathrm{g} / \mathrm{ml})$, antirTgP (various concentrations), anti-hTgP (various concentrations) or, as controls, with OVA (various concentrations), normal rabbit IgG (various concentrations) or normal human IgG (various concentrations). In the case of unlabeled rat or human $\mathrm{Tg}$, lactoferrin and OVA, biotin-labeled heparin was also pre-incubated overnight at $4^{\circ} \mathrm{C}$ with the competitors.

\section{Results}

\section{Binding of heparin to rat and human Tg}

Binding of heparin to purified human and rat $\mathrm{Tg}$ was studied in solid-phase binding assays, by measuring binding of a biotin-labeled heparin-albumin complex to microtiter wells coated with the two Tg preparations. As shown in Fig. 1A, biotin-labeled heparin bound to both rat and human $\mathrm{Tg}$ in a dose-dependent, saturable manner. In contrast, biotin-labeled albumin, used as a control, did not bind to either rat or human $\mathrm{Tg}$ (Fig. 1B). Binding of heparin to rat Tg was seen starting at $1.5 \mathrm{nmol} / \mathrm{l}$ coated protein, the lowest amount used, whereas binding to human $\mathrm{Tg}$ was seen starting at $7.5 \mathrm{nmol} / \mathrm{l}$ (Fig. 1A). The extent of binding of biotinlabeled heparin was greater for rat $\mathrm{Tg}$ than for human $\mathrm{Tg}$ at all the $\mathrm{Tg}$ concentrations used for coating. On average, the extent of binding of biotin-labeled heparin to rat $\mathrm{Tg}$ was approximately twofold greater than binding to human $\mathrm{Tg}$.

We estimated the constants of dissociation $\left(K_{\mathrm{d}}\right)$ and the $\mathrm{B}_{\max }$ of binding of biotin-labeled heparin to rat and human $\mathrm{Tg}$ at the midsaturation points (Table 2). Biotin-labeled heparin bound with a greater affinity to rat $\mathrm{Tg}\left(\right.$ mean $\left.K_{\mathrm{d}}: 34.2 \mathrm{nmol} / \mathrm{l}\right)$ than to human $\mathrm{Tg}$ $\left(\right.$ mean $\left.K_{\mathrm{d}}: 118 \mathrm{nmol} / \mathrm{l}\right)$. As expected from the greater heparin-binding capacity of rat $\mathrm{Tg}$, the $\mathrm{B}_{\max }$ was greater for rat $\mathrm{Tg}(306 \mathrm{pg})$ than for human $\mathrm{Tg}$ (241 pg). However, the higher ( 1.2-fold) $\mathrm{B}_{\max }$ (binding capacity) of rat $\mathrm{Tg}$ did not parallel its greater $(\sim 3.4$-fold $)$ binding affinity as compared with human $\mathrm{Tg}$.

We studied the effect of pre-incubation and co-incubation of biotin-labeled heparin with increasing concentrations of homologous $\mathrm{Tg}$ on its binding to the two $\mathrm{Tg}$ preparations at coating concentrations at which saturation of binding was seen (deduced from the experiments shown in Fig. 1), which were $15 \mathrm{nmol} / \mathrm{l}$ for rat Tg and $75 \mathrm{nmol} / \mathrm{l}$ for human Tg. Binding of biotin-labeled heparin to both rat (Fig. 2A) and human (Fig. 2B) Tg was inhibited in a dose-dependent, saturable manner by rat and human $\mathrm{Tg}$ themselves respectively, but not by OVA (used as a control). The extent of inhibition was much greater for rat $\mathrm{Tg}$ ( $\sim 80 \%$ at saturation) than for human $\mathrm{Tg}(\sim 25 \%$ at saturation), as expected by the lower binding affinity of human $\mathrm{Tg}$. Thus, the mean constant of inhibition $\left(K_{\mathrm{i}}\right)$, was 12 -fold lower $(37.6 \mathrm{nmol} / \mathrm{l}$, Fig. $2 \mathrm{~A})$ for rat $\mathrm{Tg}$ than for human $\mathrm{Tg}$ (480 nmol/l, Fig. 2B).

As shown in Fig. 3, binding of biotin-labeled heparin to the $\mathrm{Tg}$ preparations at coating concentrations at which saturation of binding was seen was reduced by pre-incubation and/or co-incubation of biotin-labeled heparin with several competitors, but not by OVA, used as a control. The extent of inhibition obtained was always greater for rat $\mathrm{Tg}$ than for human $\mathrm{Tg}$. Thus, unlabeled heparin reduced binding of biotinlabeled heparin by $\sim 60 \%$ to rat $\mathrm{Tg}$ and by $\sim 20 \%$ to human $\mathrm{Tg}$. Lactoferrin, a potent heparin-binding protein, reduced binding of biotin-labeled heparin to rat $\mathrm{Tg}$ by $\sim 45 \%$ and to human $\mathrm{Tg}$ by $\sim 35 \%$. The results confirm the greater binding affinity of heparin for rat $\mathrm{Tg}$ than for human $\mathrm{Tg}$.
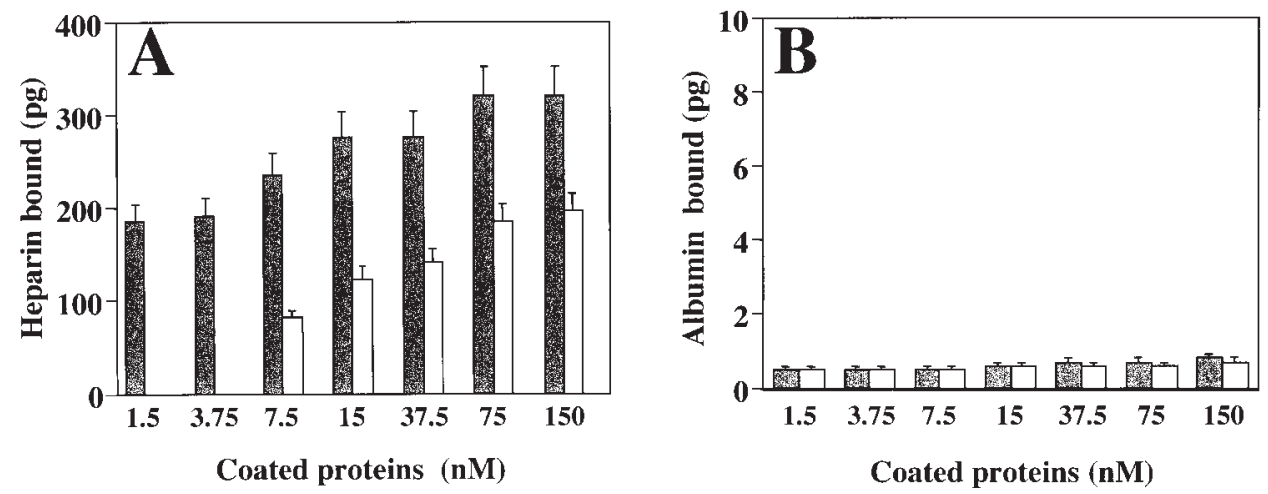

Figure 1 (A) Binding of biotin-labeled heparin to rat and human Tg. Wells coated with rat Tg (shaded bars) or human Tg (open bars) or, as a control, with OVA (all zero) were incubated with biotin-labeled heparin, followed by ALP-conjugated streptavidin and p-nitrophenylphosphate. Absorbance was determined at $405 \mathrm{~nm}$. Results are expressed as means \pm S.E. obtained in three separate experiments. (B) Absence of binding of biotin-labeled albumin to rat and human $\mathrm{Tg}$. Wells coated with rat $\mathrm{Tg}$ (shaded bars) or human $\mathrm{Tg}$ (open bars) were incubated with biotin-labeled albumin, followed by ALP-conjugated streptavidin and p-nitrophenyl-phosphate. Absorbance was determined at $405 \mathrm{~nm}$. Results are expressed as means \pm S.E. obtained in three separate experiments. 

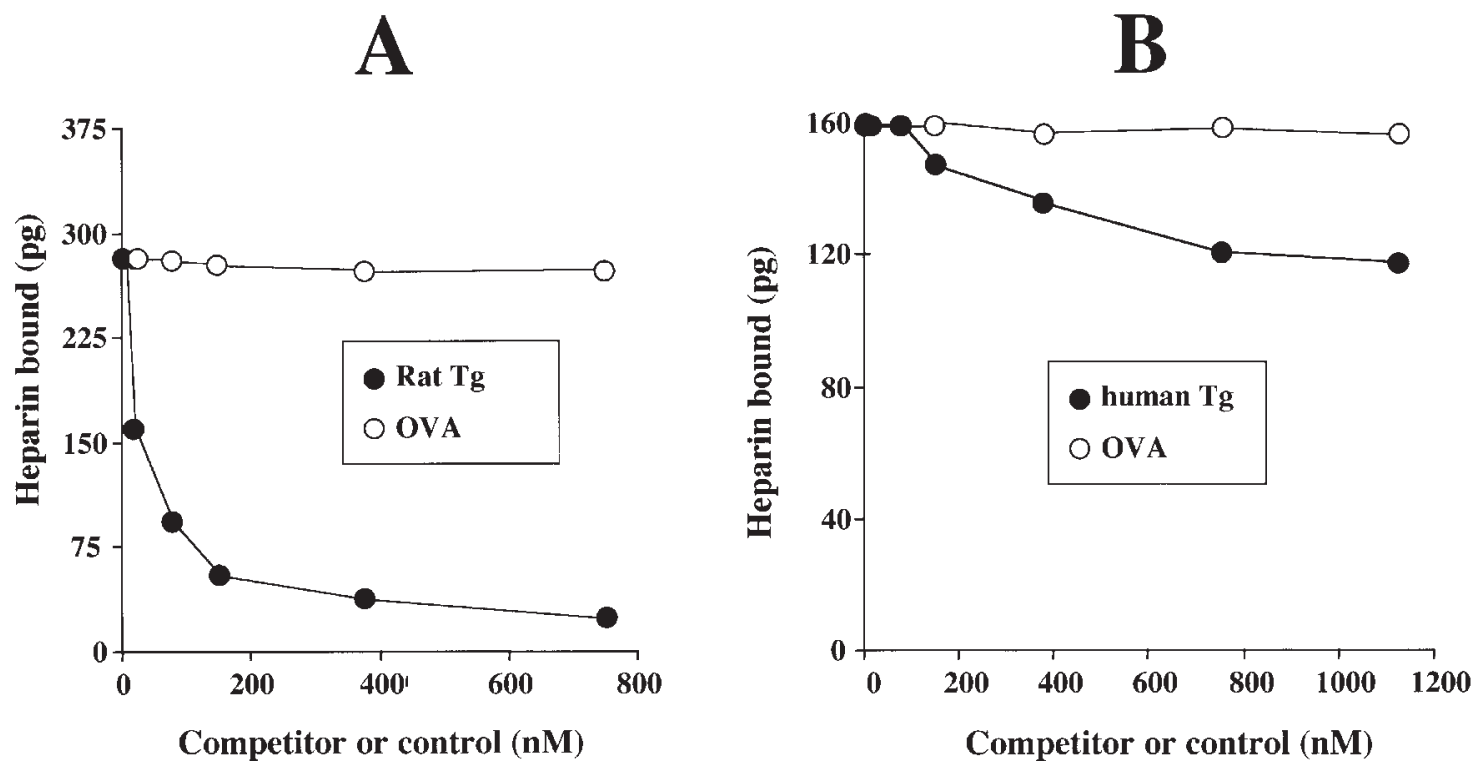

Figure 2 Inhibition of binding of biotin-labeled heparin to rat $\mathrm{Tg}$ by rat $\mathrm{Tg}$ itself $(\mathrm{A})$ and to human $\mathrm{Tg}$ by human $\mathrm{Tg}$ itself (B). Wells coated with rat $(A)$ or human $(B) T g$ were incubated with biotin-labeled heparin alone, or together with various concentrations of rat (A) or human (B) Tg, or, as a control, of OVA, after overnight pre-incubation, followed by ALP-conjugated streptavidin and p-nitrophenylphosphate. Absorbance was determined at $405 \mathrm{~nm}$. The figures are representative of one of three separate experiments.

\section{Binding of heparin to synthetic peptides}

To study if the different carboxyl terminal sequences of rat and human Tg (corresponding to the heparinbinding site of rat Tg we had identified (6)) influence the heparin-binding ability, we used a synthetic peptidebased approach. For this purpose, we used a previously described synthetic peptide designated $\mathrm{rTgP}$ and corresponding to the heparin-binding sequence of rat $\mathrm{Tg}(6)$. In addition, we used another synthetic peptide with the corresponding sequence of human $\mathrm{Tg}$ (hTgP) (Table 1). Binding of biotin-labeled heparin to rTgP- and hTgPcoated plates was studied in solid phase assays as described above.

As shown in Fig. 4, biotin-labeled heparin bound to both $\mathrm{rTgP}$ and hTgP. Binding of biotin-labeled heparin to $\mathrm{rTgP}$ was seen starting at $1.25 \mu \mathrm{mol} / \mathrm{l}$ coated peptide, the lowest amount used, whereas binding to $\mathrm{hTgP}$ was seen starting at $7.5 \mu \mathrm{mol} / \mathrm{l}$, a behavior similar to that observed for binding of heparin to rat and human $\mathrm{Tg}$, as described above and in Fig. 1.

Table 2 Binding of biotin-labeled heparin to $\mathrm{Tg}$ preparations: mean constants of dissociation $\left(K_{\mathrm{d}}\right)$ and $B_{\max }$.

\begin{tabular}{lcc}
\hline & $\boldsymbol{K}_{\mathbf{d}}(\mathrm{nmol} / \mathrm{l})$ & $\mathbf{B}_{\mathbf{m a x}}(\mathrm{pg})$ \\
\hline Rat $\mathrm{Tg}$ & 34.2 & 306 \\
Human Tg & 118 & 241 \\
\hline
\end{tabular}

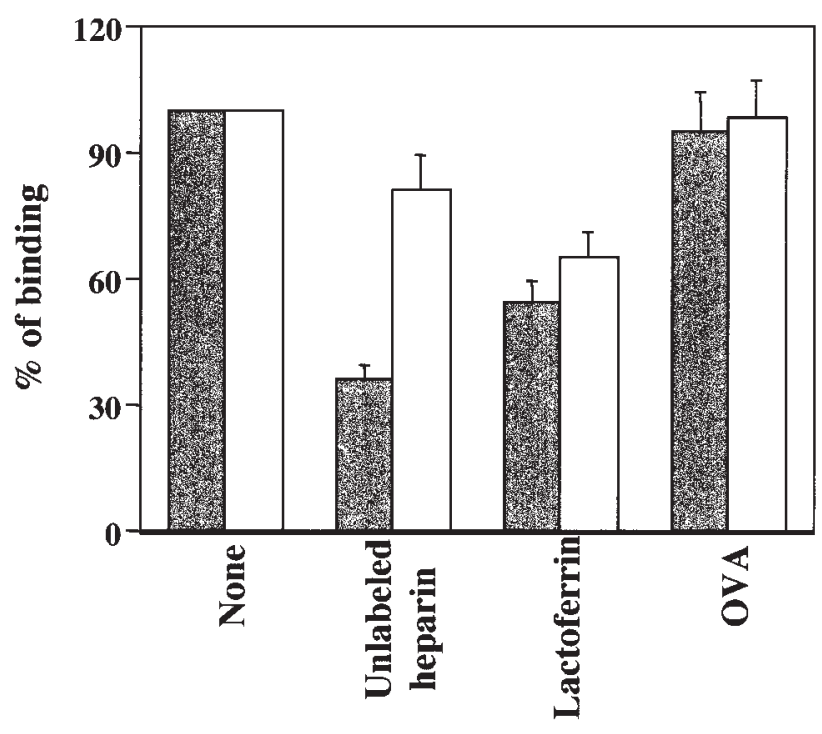

\section{Competitor or control}

Figure 3 Inhibition of binding of biotin-labeled heparin to rat $\mathrm{Tg}$ (shaded bars) or to human Tg (open bars) by unlabeled heparin or lactoferrin. Wells coated with rat or human Tg were incubated with biotin-labeled heparin alone, or together with unlabeled heparin, lactoferrin (after overnight pre-incubation) or, as a control, with OVA (after overnight pre-incubation), followed by ALP-

conjugated streptavidin and $p$-nitrophenyl-phosphate. Absorbance was determined at $405 \mathrm{~nm}$. Results are expressed as mean \pm S.E. percentage of binding obtained in three separate experiments. 


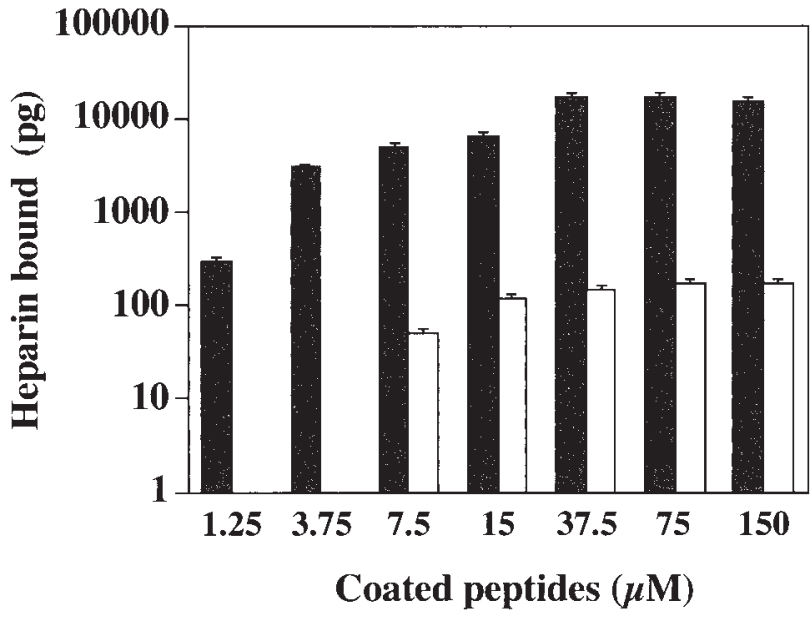

Figure 4 Binding of biotin-labeled heparin to synthetic peptides. Wells coated with rTgP (shaded bars) or hTgP (open bars) were incubated with biotin-labeled heparin, followed by ALP-conjugated streptavidin and p-nitrophenyl-phosphate. Absorbance was determined at $405 \mathrm{~nm}$. Results are expressed as means \pm S.E. obtained in three separate experiments.

Biotin-labeled heparin bound to rTgP to a greater extent than to hTgP at all the peptide concentrations used for coating. On average, the extent of binding of biotin-labeled heparin to rTgP was approximately 100-fold greater than binding to hTgP.

We estimated the $K_{\mathrm{d}}$ of binding of biotin-labeled heparin to the two peptides (Table 3). The estimates indicate that biotin-labeled heparin bound with a greater affinity to $\mathrm{rTgP}$ (mean $K_{\mathrm{d}}: 15 \mu \mathrm{mol} / \mathrm{l}$ ) than to hTgP (mean $\left.K_{\mathrm{d}}: 56 \mu \mathrm{mol} / \mathrm{l}\right)$. Thus, the binding affinity was about threefold greater for rTgP. The apparent greater extent of binding of biotin-labeled heparin to the rTgP (Fig. 4) than to rat Tg (Fig. 1) can be explained by the much greater molar amounts of coated peptide used $(1.5-150 \mu \mathrm{mol} / \mathrm{l})$ than of coated rat $\mathrm{Tg}(1.5-150 \mathrm{nmol} / \mathrm{l})$.

The results indicate that the human $\mathrm{Tg}$ sequence Arg2489-Glu2503 exhibits heparin binding, although to a lower extent and with lower affinity than the corresponding sequence of rat $\mathrm{Tg}$.

\section{Mutational study}

The sequences of rat and human $\mathrm{Tg}$ in the carboxyl terminal heparin-binding site differ by six amino acid residues (Table 1 ). In an attempt to investigate which of the six residues are responsible for the lower heparin-binding ability of the human $\mathrm{Tg}$ sequence, we used 6 synthetic 'mutant' peptides (Table 1). Starting from the rat $\mathrm{Tg}$ sequence, each mutant peptide was designed by replacing one amino acid residue with the corresponding residue of the human $\mathrm{Tg}$ sequence. The peptides were designated mutants 5 to 10 (mutants 1 to 4 had been used in a previous study (6)).
Table 3 Binding of biotin-labeled heparin to synthetic pepides: mean constants of dissociation $\left(K_{\mathrm{d}}\right)$.

\begin{tabular}{lc}
\hline Peptide & $\boldsymbol{K}_{\mathbf{d}}(\mu \mathrm{mol} / \mathrm{l})$ \\
\hline $\mathrm{rTgP}$ & 15 \\
hTgP & 56 \\
Mutant 5 & 80 \\
Mutant 6 & 50 \\
Mutant 7 & 92.5 \\
Mutant 8 & 75 \\
Mutant 9 & 19 \\
Mutant 10 & 75 \\
\hline
\end{tabular}

We tested binding of biotin-labeled heparin to wells coated with the 6 mutant peptides in solid phase binding assays, as compared with binding to rTgP and hTgP. As shown in Fig. 5 and in Table 3, biotin-labeled heparin bound to the mutant peptides to a lower extent and with lower affinity than to the rTgP, with the exception of mutant 9. Thus, as shown in Table 3, $K_{\mathrm{d}}$ values of binding of biotin-labeled heparin to mutants 5, 6, 7, 8 and 10 were three- to sixfold higher than the $K_{\mathrm{d}}$ value of binding of biotin-labeled heparin to rTgP. In contrast, biotin-labeled heparin bound to mutant 9 to a similar extent and with similar affinity $\left(K_{\mathrm{d}}: 19 \mu \mathrm{mol} / \mathrm{l}\right)$ as to $\mathrm{rTgP}$. As compared with hTgP, heparin bound to the mutant peptides to an overall similar extent and with similar affinity, with the exception of mutant 9 to which it clearly bound to a greater extent and with greater affinity.

The results suggest that the greater binding capacity of rTgP may depend not only on charge, but also on sequence and/or conformation, because not only substitution of positively charged amino acid residues, as we found here and in our previous study (6), but also substitution of neutral residues, markedly reduced its heparin-binding capacity.

\section{Purification and characterization of a human polyclonal antibody against hTgP}

In order to prepare an antibody against hTgP we took advantage of the knowledge that hTgP (amino acids (aa) 2489-2503) overlaps an immunogenic epitope of $\mathrm{Tg}$ (aa 2495-2511) that is capable of inducing an experimental autoimmune thyroiditis in rats $(26,27)$. With the aim of purifying serum IgGs directed against hTgP, we first investigated whether anti-Tg autoantibodies in sera from patients with autoimmune thyroiditis are capable of reacting with hTgP. For this purpose, we used sera from eleven patients with autoimmune thyroiditis, ten of which had been found to contain autoantibodies against Tg. We tested the reactivity of IgGs in these sera against hTgP by ELISA. As shown in Fig. 6A, IgGs in 6 of 10 anti-Tg-positive sera clearly reacted with hTgP (numbers 1 and $6-10$ ), whereas IgGs in the remaining four sera with anti-Tg autoantibodies 


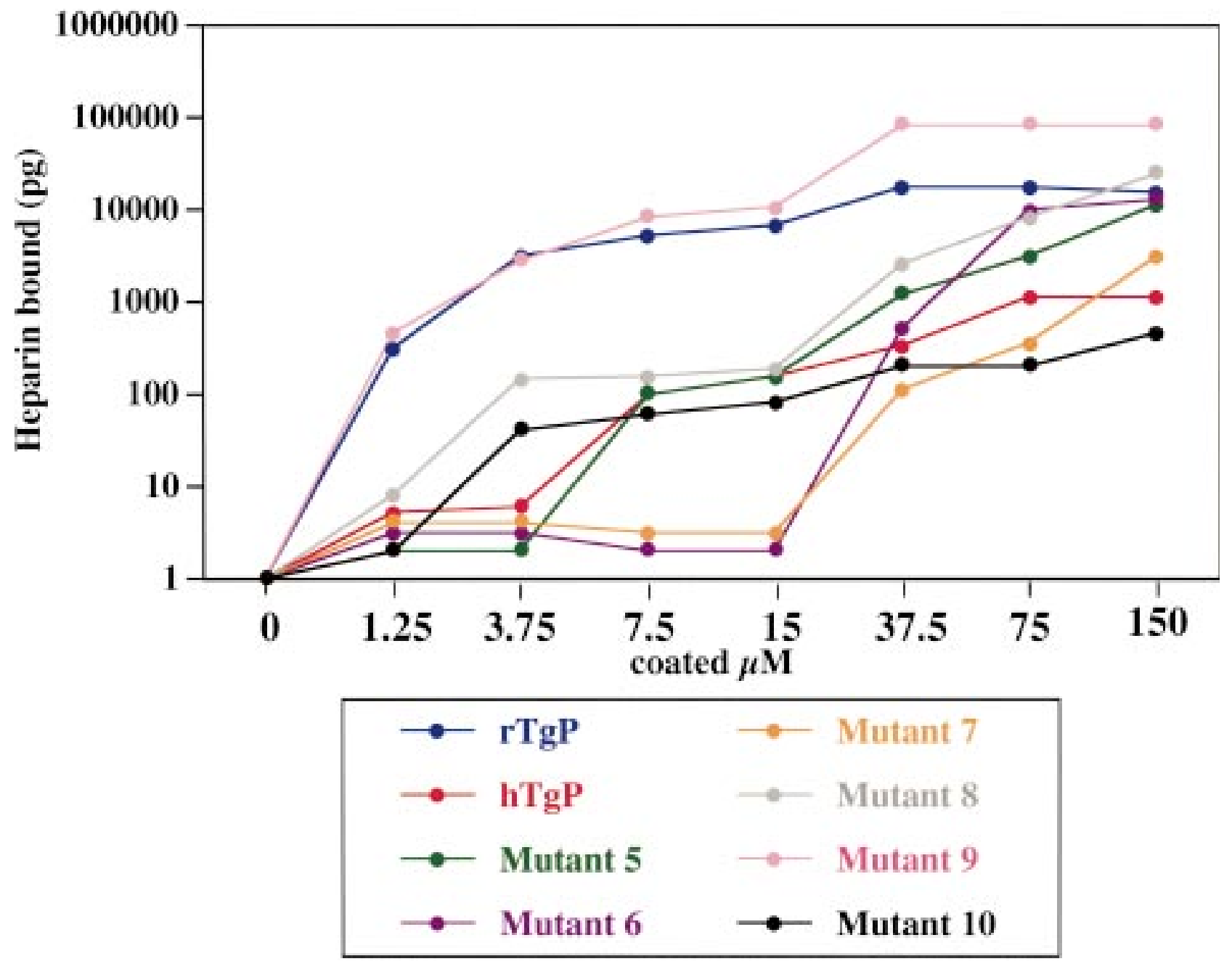

Figure 5 Binding of biotin-labeled heparin to synthetic peptides. Wells coated with $\mathrm{rTgP}, \mathrm{hTgP}$ or with six mutant peptides were incubated with biotin-labeled heparin, followed by ALP-conjugated streptavidin and p-nitrophenyl-phosphate. Absorbance was determined at $405 \mathrm{~nm}$. The figure is representative of one of three separate experiments.

as well as in the serum without anti-Tg autoantibodies did not (numbers 2-5). Three of the six positive sera (sera 6, 7 and 10) were used for further experiments because they were available in larger quantities. As shown in Fig. 6B, binding of IgGs to hTgP in sera 6 , 7 and 10 was reduced by $\sim 30$ to $\sim 60 \%$ by pre-incubation and co-incubation of sera with hTgP itself, indicating that binding was specific. No effect on binding of IgGs to hTgP was produced by co-incubation and preincubation of sera with a control peptide (Table 1), used as a negative control.

An antibody against hTgP was then immunoaffinity purified from a pool of sera 6,7 and 10 , as detailed in the Materials and methods section. The reactivity of the anti-hTgP antibody against hTgP and intact human $\mathrm{Tg}$ was tested by ELISA. As shown in Fig. 7, the antibody reacted with $\mathrm{hTgP}$ and to a greater extent with intact human $\mathrm{Tg}$, probably reflecting the existence of conformational epitopes of human $\mathrm{Tg}$ that are not entirely conserved in hTgP. No significant reactivity was found against a control peptide or against BSA used as controls.

\section{Effect of anti-rTgP and anti-hTgP antibodies on heparin binding to $\mathbf{T g}$}

In a previous study (6) we demonstrated that a rabbit antibody against rTgP markedly reduced binding of heparin to rat $\mathrm{Tg}$, providing evidence that the rat $\mathrm{Tg}$ sequence corresponding to $\mathrm{rTgP}$ represents a major binding site for heparin. In confirmation of our previous study (6), here we found in solid-phase binding assays that co-incubation of biotin-labeled heparin with the anti-rTgP antibody reduced its binding to intact $\mathrm{Tg}$ in a concentration-dependent, saturable manner, reaching $70 \%$ inhibition of binding at saturation (Fig. 8A). No effect was produced by normal rabbit IgG used as a control. 


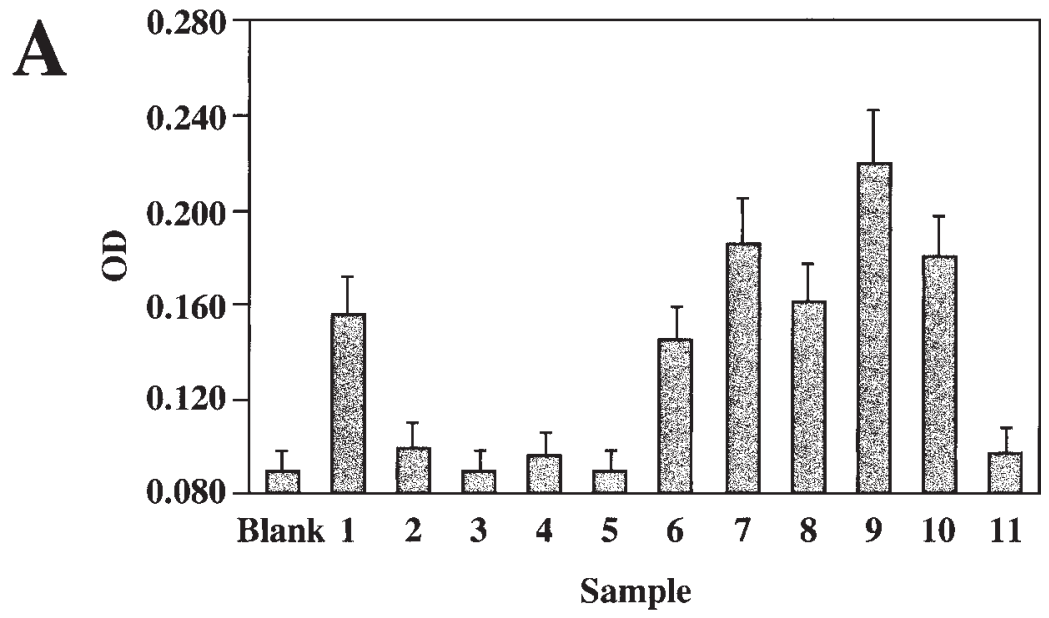

B

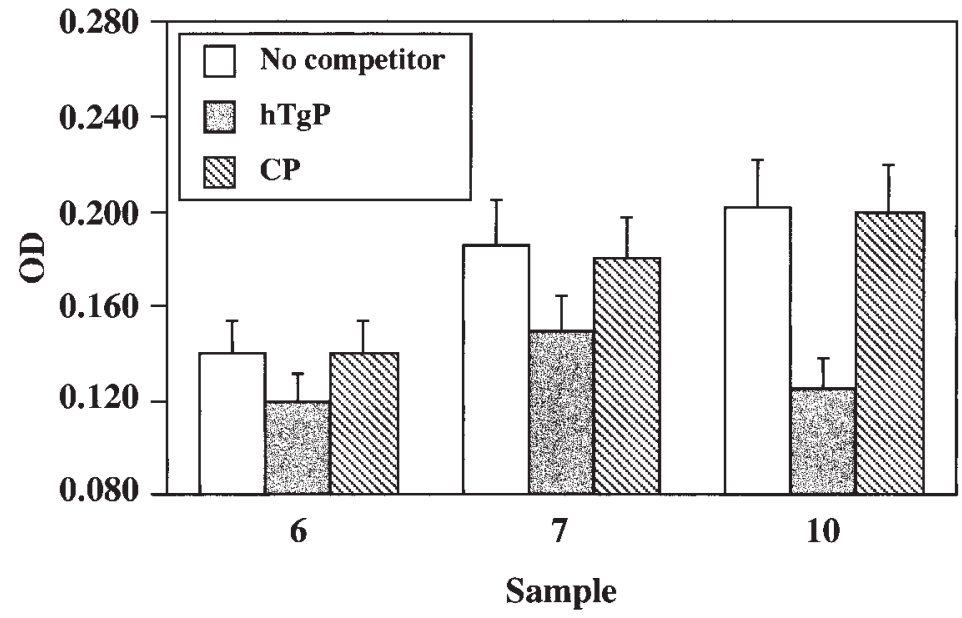

Figure 6 (A) Selection of human sera containing autoantibodies against hTgP. Wells coated with $\mathrm{hTgP}$ were incubated with sera from eleven patients with autoimmune thyroiditis, numbered from 1 to 11 , followed by ALP-conjugated anti-human IgG and by p-nitrophenyl-phosphate. Absorbance was determined at $405 \mathrm{~nm}$. Results are expressed as means \pm S.E. obtained in three separate experiments. (B) Inhibition of binding of IgGs in sera 6,7 and 10 to hTgP. Wells coated with $\mathrm{hTgP}$ were incubated with sera alone, or in the presence of hTgP or, as a control, with a control peptide (CP), followed by ALP-conjugated anti-human IgG and by p-nitrophenylphosphate. Absorbance was determined at $405 \mathrm{~nm}$. Results are expressed as means \pm S.E. obtained in three separate experiments. OD, optical density.

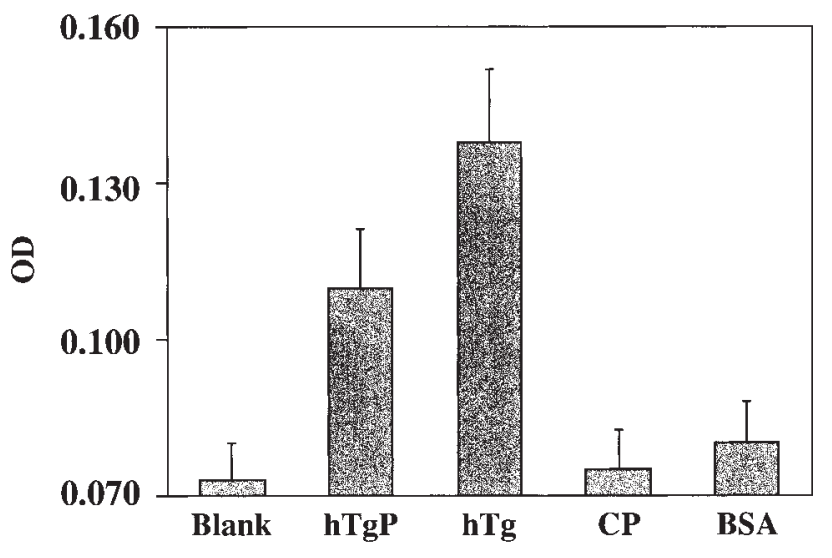

Figure 7 Binding of an antibody purified from sera 6,7 and 10 (see Fig. 6) to hTgP and to intact human Tg. Wells, either uncoated (blank) or coated with hTgP, intact human Tg, or, as controls, with a control peptide (CP) or with BSA, were incubated with the purified antibody followed by ALP-conjugated anti-human IgG and by $p$-nitrophenyl-phosphate. Absorbance was determined at $405 \mathrm{~nm}$. Results are expressed as means \pm S.E. obtained in three separate experiments. OD, optical density.
We investigated the effect of the anti-hTgP antibody on binding of biotin-labeled heparin to intact human $\mathrm{Tg}$ in solid phase binding assays. As shown in Fig. 8B, co-incubation of biotin-labeled heparin with the anti-hTgP antibody reduced its binding to human $\mathrm{Tg}$ in a concentration-dependent, saturable manner, with an inhibition of $30 \%$ at saturation. The results suggest that the human $\mathrm{Tg}$ region corresponding to $\mathrm{hTgP}$ represents a heparin-binding site which, however, contributes to total binding of heparin to a lower extent than the corresponding region of rat $\mathrm{Tg}$. Thus, the contribution of other heparin-binding site/s is greater in human $\mathrm{Tg}$ than in rat $\mathrm{Tg}$.

\section{Discussion}

The present study was undertaken to investigate the heparin-binding ability of human $\mathrm{Tg}$ as compared with rat Tg. Our investigations stemmed from the knowledge that the major heparin-binding site of rat Tg Arg2489-Lys2503 (RELPSRRLKRPLPVK), located 


\section{A}

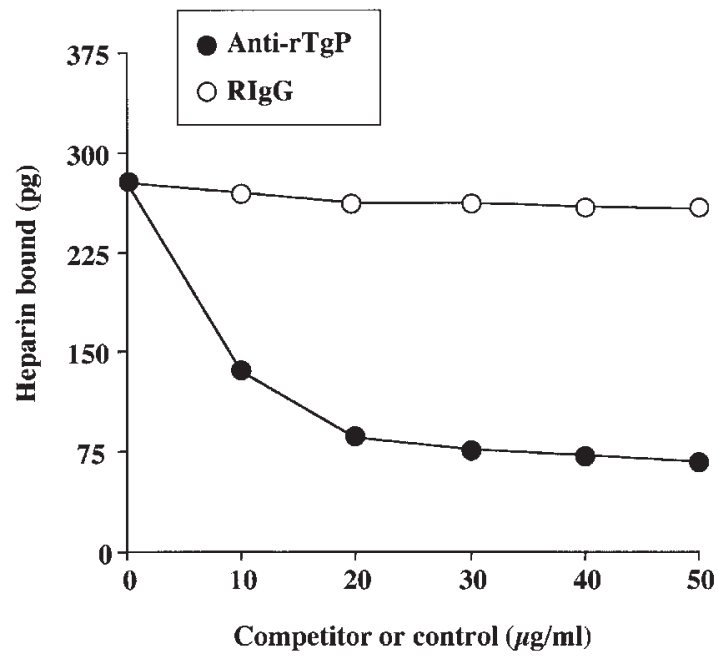

B

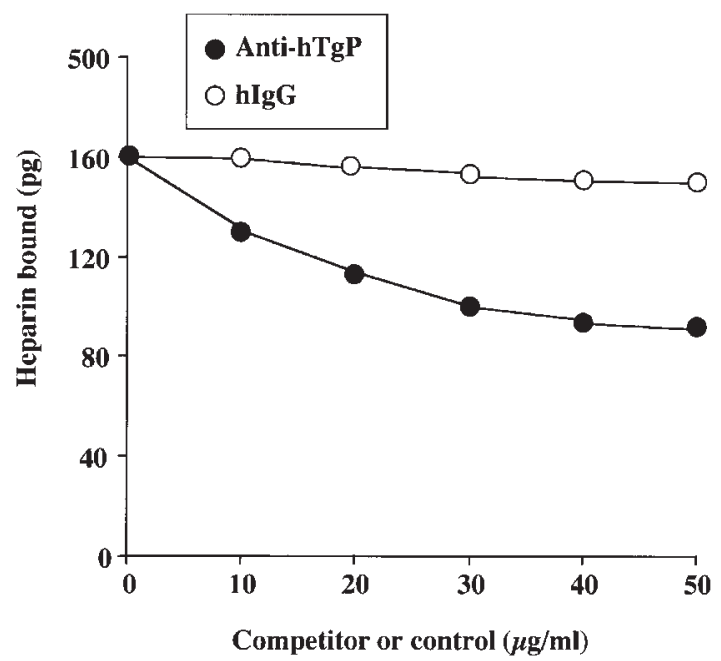

Figure 8 Inhibition of binding of biotin-labeled heparin to rat (A) or to human (B) Tg by antibodies directed against a carboxyl terminal heparin-binding site. Wells coated with rat $(A)$ or human $(B)$ Tg were incubated with biotin-labeled heparin alone, or together with various concentrations of rabbit anti-rTgP $(\mathrm{A})$ or of human anti-hTgP $(\mathrm{B})$, or, as controls, with normal rabbit $(\mathrm{A}, \mathrm{RlgG})$ or human (B, hlgG) IgG, followed by ALP-conjugated streptavidin and p-nitrophenyl-phosphate. Absorbance was determined at $405 \mathrm{~nm}$. The figures are representative of one of three separate experiments.

in the carboxyl terminal portion of the molecule (22), is not entirely conserved in human $\mathrm{Tg}$ (Arg2489Glu2503, REPPARALKRSLWVE) (24). In spite of this difference, we found that human $\mathrm{Tg}$ is capable of heparin binding, although to a lower extent and with lower affinity than rat Tg, using alternative, as yet unidentified, heparin-binding sites, in addition to the carboxyl terminal heparin-binding site. Results supporting these conclusions can be summarized as follows.

In confirmation of previous studies (6), biotin-labeled heparin bound to rat $\mathrm{Tg}$ in solid-phase binding assays with moderately high affinity $\left(K_{\mathrm{d}}: 34.2 \mathrm{nmol} / \mathrm{l}, K_{\mathrm{i}}\right.$ : $37.6 \mathrm{nmol} / \mathrm{l})$. Here we found that biotin-labeled heparin bound to human $\mathrm{Tg}$, but with lower affinity $\left(K_{\mathrm{d}}\right.$ : $\left.118 \mathrm{nmol} / \mathrm{l}, \quad K_{\mathrm{i}}: 480 \mathrm{nmol} / \mathrm{l}\right)$ and to a lower extent than to rat Tg. Binding to both Tg preparations was dose-dependent and saturable, and was reduced by several specific competitors, including homologous $\mathrm{Tg}$ itself, unlabeled heparin and lactoferrin, a potent heparin-binding protein. The extent of inhibition was greater for rat $\mathrm{Tg}$ than for human $\mathrm{Tg}$, as expected from the greater heparin-binding affinity of rat Tg.

The carboxyl terminal sequence of human $\mathrm{Tg}$ Arg2489-Glu2503 was found to be, in part, responsible for heparin binding. Thus, biotin-labeled heparin bound to a synthetic peptide (hTgP) corresponding to this sequence, although to a lower extent and with lower affinity than to a peptide carrying the corresponding sequence of rat $\mathrm{Tg}(\mathrm{rTgP})$. Furthermore, a natural polyclonal human autoantibody capable of recognizing intact human $\mathrm{Tg}$ as well as hTgP reduced binding of heparin to intact human $\mathrm{Tg}$.

In an attempt to investigate how the differences in the carboxyl terminal heparin-binding sequences of rat and human $\mathrm{Tg}$ affect the heparin-binding ability of this region, we performed a mutational study. We designed 6 synthetic 'mutant' peptides by replacing one amino acid residue of $\mathrm{rTgP}$ with the corresponding residue of the human $\mathrm{Tg}$ sequence. Biotin-labeled heparin bound to 5 of the 6 mutant peptides to a lower extent and with lower affinity than to rTgP. Because not only substitution of positively charged amino acid residues, as we found here and in our previous study (6), but also substitution of neutral residues reduced the heparin-binding ability of $\mathrm{rTgP}$, we postulate that not only charge, but also sequence and/or conformation may account for the heparin-binding ability of this region of $\mathrm{Tg}$. Results obtained with mutants 5 and 9 suggest that sequence may be more important than conformation in the case of mutant 9. In both these mutants neutral amino acid residues were substituted with other neutral residues. In mutant 5 substitution of leucine with proline reduced the heparin-binding ability, whereas in mutant 9 substitution of proline with tryptophan had virtually no effect. Because proline is known to be more prone to affect conformation than tryptophan, if the heparinbinding ability were entirely dependent on conformation, a reduced binding would have been expected in mutant 9 where proline was replaced by tryptophan. 
Thus, replacement of proline with another neutral amino acid residue (tryptophan) in mutant 9 should have affected conformation as did replacement of leucine with proline in mutant 5 . Nevertheless, whether sequence or conformation or both are responsible for heparin binding and how they contribute to the heparin-binding ability of this $\mathrm{Tg}$ region is unknown. Further studies are needed to clarify these issues, aimed at investigating whether and how the possibly different conformational modes of the various Tg peptides affect their heparin-binding ability, after their three-dimensional structure is discovered through crystallization studies. In addition, further studies using peptides carrying simultaneously more than one mutation may also be useful in clarifying these issues.

As mentioned above, in a previous study we demonstrated that the carboxyl terminal rat $\mathrm{Tg}$ sequence Arg2489-Lys2503 represents a major heparin-binding site (6). In addition, this sequence is required for rat $\mathrm{Tg}$ binding to HSPGs (9). The fact that a rabbit antibody raised against Arg2489-Lys2503 (rTgP) reduced heparin-binding to rat $\mathrm{Tg}$ by only $70 \%$, led us to the conclusion that other heparin-binding sites must account, in part, for binding (6). However, although by analysis of the rat $\mathrm{Tg}$ sequence we identified an alternative potential heparin-binding site in another region of $\mathrm{Tg}$, we failed to demonstrate its involvement in heparin-binding (6). Therefore, we concluded that, most likely, additional heparin-binding sites must correspond to clusters of positively charged amino acids, distant in the sequence, but brought together by the folding of the protein.

Several considerations indicate that the corresponding carboxyl terminal sequence of human $\mathrm{Tg}$ Arg2489-Glu2503 contributes only minimally to its overall heparin-binding ability, and that other regions must be involved to a greater extent, in contrast with rat $\mathrm{Tg}$. First, the lower heparin-binding capacity of human $\mathrm{Tg}$ did not parallel linearly its lower binding affinity as compared with rat $\mathrm{Tg}$, indicating that human $\mathrm{Tg}$ can compensate for a lower heparin-binding affinity with the presence of multiple heparin-binding sites. Secondly, the lower heparin-binding affinity of hTgP compared with rTgP did not parallel linearly the difference between human and rat $\mathrm{Tg}$; it was in fact much lower: 100-fold vs 3.4-fold. This finding indicates that the contribution of the region of human $\mathrm{Tg}$ corresponding to hTgP to total heparin-binding must be rather low, unless the conformation of this region in the intact molecule differs from the conformation of hTgP in a way that allows a greater heparin-binding affinity. Thirdly, and more importantly, the anti-hTgP antibody reduced binding of biotin-labeled heparin to intact human $\mathrm{Tg}$ by only $30 \%$ at saturation whereas, as mentioned above, the corresponding region of rat $\mathrm{Tg}$ contributes $70 \%$ to heparin-binding.

By analysis of the sequence of human Tg (24), we did not find any putative heparin-binding sites (Cardin and
Weintraub's motifs). Therefore, as in the case of rat $\mathrm{Tg}$, we concluded that other heparin-binding sites must correspond to clusters of positively charged amino acid residues, distant in the sequence but brought together by the folding of the protein. The optimal way to study how conformation affects the heparinbinding ability of a protein is to analyze its three-dimensional structure, to locate regions rich in positively charged residues and to determine whether they are brought together by the folding of the protein $(2-3)$. However, this approach is not currently possible in the case of $\mathrm{Tg}$, because its three-dimensional structure is not known. Another way to study putative heparinbinding sites is by site-directed mutagenesis studies (28). However, this approach is unlikely to be feasible in the case of Tg. Thus, although Kim et al. (29) have succeeded in transfecting a nearly full length Tg gene in cultured cells, point mutations in the carboxyl-terminal portion abolished secretion of the protein, thereby making it impossible to obtain preparations of purified mutated Tg.

The heparin-binding ability of proteins allows their interaction with GAGs $(2-4)$. In the case of rat $\mathrm{Tg}$, binding to HSPGs allows optimal binding to its endocytic receptor, megalin (9). Further studies are needed to investigate whether and how the difference in the sequence of human $\mathrm{Tg}$ and its different heparin-binding capacity affect its binding to HSPGs. Recently, SiffroiFernandez et al. (30) provided evidence that the human $\mathrm{Tg}$ carboxyl terminal region from Ser2445 to Met2596, comprising the sequence we studied here (Arg2489-Glu2503), is involved in low affinity Tg binding to as yet unidentified molecules on thyroid cell membranes. Based on the results presented here it is worth considering the possibility that binding may be due to HSPGs.

$\mathrm{Tg}$ is thought to be present in the colloid in a variety of folding modes and a relevant proportion of $\mathrm{Tg}$ in the colloid is multimerized and insoluble and undergoes partial proteolysis $(31-40)$. Therefore, it is possible that not all the $\mathrm{Tg}$ forms present in the colloid display the same behavior concerning heparin binding and endocytosis, and certain forms may bind to HSPGs and to $\mathrm{Tg}$ receptors to a greater extent than others. Because, as mentioned above, the three-dimensional structure of $\mathrm{Tg}$ is unknown, it is impossible at the moment to study directly how folding of Tg affects its heparin-binding ability. The $\mathrm{Tg}$ preparations used in the present study corresponded exclusively to the soluble forms of $\mathrm{Tg}$ as, during $\mathrm{Tg}$ purification, the insoluble forms are commonly discarded. Therefore, whether our results apply to multimerized $\mathrm{Tg}$ or to $\mathrm{Tg}$ that has undergone partial proteolysis is unknown and requires further investigation. Nevertheless, results presented here are relevant for understanding the mechanisms of $\mathrm{Tg}$ binding to heparinoids in the general context of $\mathrm{Tg}$ endocytosis and hormone release. Thus, under physiological conditions the forms of Tg that undergo 
hormone release following endocytosis are soluble and have not undergone proteolysis prior of endocytosis, as they are thought to be the first secreted by thyrocytes after their synthesis (last come first served theory) (41). In this view, as we have shown in previous studies $(7,9)$, the Tg that is endocytosed by the receptor megalin - a process facilitated by Tg binding to HSPGs and that is involved in the regulation of hormone release is soluble, intact and hormonogenic, as were the $\mathrm{Tg}$ preparations used in the present study (not shown).

The difference in the heparin-binding ability between human and rat $\mathrm{Tg}$ may represent the consequence of an evolutionary adaptation of the thyroid to the necessity of a high metabolic rate in rodents. Binding to HSPGs may render rat $\mathrm{Tg}$ uptake and hormone release more efficient than in larger mammals, resulting in a higher metabolic rate. Further studies are needed to investigate these issues.

\section{Acknowledgements}

This work was supported by the American Thyroid Association Research grant (to M M), by grants from the National Research Council (Consiglio Nazionale Ricerche, Roma, Italy) (grants 91.01219 and 9300437, to AP and LC), by EEC Stimulation Action-Science Plan (contract SC1-CT91-0707, to AP and L C) and by a grant from the Italian Association for Cancer Research (AIRC, to M M).

\section{References}

1 Cardin AD \& Weintraub HJR. Molecular modeling of proteinglycosaminoglycan interactions. Atherosclerosis 1989 9 21-32.

2 Conrad HE. Heparin-binding proteins. In Heparin-Binding Proteins, edn 1, ch. 3, pp 183-202. Ed. HE Conrad. San Diego: Academic Press, 1998.

3 Conrad HE. Heparin-binding proteins in lipoprotein metabolism. In Heparin-Binding Proteins, edn 1, ch. 6, pp 367-409. Ed. HE Conrad. San Diego: Academic Press, 1998.

4 Conrad HE. Epilog. In Heparin-Binding Proteins, edn 1, ch. 9, pp 413-415. Ed. HE Conrad. San Diego: Academic Press, 1998.

5 Marinò M, Zheng G \& McCluskey RT. Megalin (gp330) is an endocytic receptor for thyroglobulin on cultured fisher rat thyroid cells. Journal of Biological Chemistry $199927412898-12904$.

6 Marinò M, Friedlander JA, McCluskey RT \& Andrews D. Identification of a heparin-binding region of rat thyroglobulin involved in megalin binding. Journal of Biological Chemistry 1999274 30377-30386.

7 Marinò M, Zheng G, Chiovato L, Pinchera A, Brown D, Andrews D et al. Role of megalin (gp330) in transcytosis of thyroglobulin by thyroid cells: a novel function in the control of thyroid hormone release. Journal of Biological Chemistry 2000275 7125-7138.

8 Marinò M, Chiovato L, Latrofa F, Andrews D, Collins BA, Mitsiades $\mathrm{N}$ et al. Circulating thyroglobulin derived from transcytosis is combined with a secretory component of its endocytic receptor megalin. Journal of Clinical Endocrinology and Metabolism 2000 853458 - 3467.

9 Marinò M, Andrews D \& McCluskey RT. Binding of thyroglobulin to heparan sulfate proteoglycans. Thyroid $200010551-559$.
10 Marinò M \& McCluskey RT. Role of thyroglobulin endocytic pathways in the control of thyroid hormone release. American Journal of Physiology Cell Physiology 2000279 C1295-C1306.

11 Marinò M, Pinchera A, McCluskey RT \& Chiovato L. Megalin in thyroid physiology and pathology. Thyroid $20001147-56$.

12 Hatipoglu BA \& Schneider AB. Selective endocytosis of thyroglobulin. A review of potential mechanisms for protecting newly synthesized molecules from premature degradation. Biochimie $199981549-555$.

13 Rousset B \& Mornex R. The thyroid hormone secretory pathway current dogmas and alternative hypotheses. Molecular and Cellular Endocrinology 199178 89-93.

14 Raychowdhury R, Niles JL, McCluskey RT \& Smith JA. Autoimmune target in Heymann nephritis is a glycoprotein with homology to the LDL receptor. Science $1989 \mathbf{2 4 4} 1163-1165$.

15 Saito A, Pietromonaco S, Loo AKC \& Farquhar MG. Complete cloning and sequencing of rat gp330/megalin, a distinctive member of the low density lipoprotein receptor gene family. PNAS $1994919725-9729$.

16 Krieger M \& Hertz J. Structures and functions of multiligand lipoprotein receptors: macrophage scavenger receptors and LDL receptor-related protein (LRP). Annual Reviews of Biochemistry $199463601-637$.

17 Hussain MM, Strickland DK \& Bakillah A. The mammalian lowdensity lipoprotein receptor family. Annual Reviews of Nutrition $199919141-172$.

18 Willnow TE, Nykjaer A \& Herz J. Lipoprotein receptors: new roles for ancient proteins. Nature Cell Biology 19991 E157-E162.

19 Zheng G, Bachinsky DR, Stamenkovic I, Strickland DK, Brown D, Andres $G$ et al. Organ distribution in rats of two members of the low-density lipoprotein receptor gene family, gp330 and $\mathrm{LRP} / \mathrm{a} 2 \mathrm{MR}$, and the receptor-associated protein (RAP). Journal of Histochemistry and Cytochemistry $1994 \mathbf{4 2} 531-542$.

20 Lundgren S, Carling T, Hjalm G, Juhlin C, Rastad J, Pihlgren et al. Tissue distribution of human gp330/megalin, a putative $\mathrm{Ca}^{2+}$. sensing protein. Journal of Histochemistry and Cytochemistry $199745383-392$.

21 Zheng G, Marinò M, Zhao J \& McCluskey RT. Megalin (gp330): a putative endocytic receptor for thyroglobulin $(\mathrm{Tg})$. Endocrinology $19981391462-1465$

22 Kim PS, Ding M, Menon S, Jung C-G, Cheng J-M, Miyamoto T et al. A missense mutation G2320R in the thyroglobulin gene causes non-goitrous congenital primary hypothyroidism in the WICrdw rat. Molecular Endocrinology 200014 1944-1953.

23 Caturegli P, Vidalain PO, Vali M, Aguilera-Galaviz LA \& Rose NR. Cloning and characterization of murine thyroglobulin cDNA. Clinical Immunology and Immunopathology $1997 \mathbf{8 5} 221-226$.

24 Malthiery Y \& Lissitzky S. Primary structure of human thyroglobulin deduced from the sequence of its 8448-base complementary DNA. European Journal of Biochemistry $1987165491-498$.

25 Marcocci C, Vitti P, Cetani F, Catalano F, Concetti R \& Pinchera A. Thyroid ultrasonography helps to identify patients with diffuse lymphocytic thyroiditis who are prone to develop hypothyroidism. Journal of Clinical Endocrinology and Metabolism $1991 \mathbf{7 2}$ 209-214.

26 Balasa B \& Carayanniotis G. Immunotargeting of thyroglobulin on antigen presenting cells abrogates natural tolerance in the absence of adjuvant. Cell Immunology $1993148259-268$.

27 Chronopoulou E \& Carayanniotis G. Identification of a thyroiditogenic sequence within the thyroglobulin molecule. Journal of Immunology 1992149 1039-1044.

28 Wong P, Hampton B, Szylobryt E, Gallagher AM, Jaye M \& Burgess WH. Analysis of putative heparin-binding domains of fibroblast growth factor-1. Using site-directed mutagenesis and peptide analogues. Journal of Biological Chemistry $199527025805-25811$.

29 Kim PS, Hossain SA, Park YN, Lee I, Yoo SE \& Arvan P. A single amino acid change in the acetylcholinesterase-like domain of thyroglobulin causes congenital goiter with hypothyroidism in the $\operatorname{cog} / \operatorname{cog}$ mouse: a model of human endoplasmic reticulumstorage diseases. PNAS 199895 9909-9913. 
30 Siffroi-Fernandez S, Delom F, Nlend M-C, Lanet J, Franc J-L \& Giraud A. Identification of thyroglobulin domain(s) involved in cell-surface binding and endocytosis. Journal of Endocrinology $2001170217-226$.

31 Brix K, Linke M, Tepel C \& Herzog V. Cysteine proteinases mediate extracellular prohormone processing in the thyroid. Biologica Chemistry 2001382 717-725.

32 Hitzel C, Kanzler H, Konig A, Kummer MP, Brix K, Herzog V et al. Thyroglobulin type-I-like domains in invariant chain fusion proteins mediate resistance to cathepsin L digestion. FEBS Letters $2000 \mathbf{4 8 5} 67-70$.

33 Tepel C, Bromme D, Herzog V \& Brix K. Cathepsin K in thyroid epithelial cells: sequence, localization and possible function in extracellular proteolysis of thyroglobulin. Journal of Cell Science $20001134487-4498$.

34 Klein M, Gestmann I, Berndorfer U, Schmitz A \& Herzog V. The thioredoxin boxes of thyroglobulin: possible implications for intermolecular disulfide bond formation in the follicle lumen. Biological Chemistry $2000 \mathbf{8 1} 593-601$.

35 Saber-Lichtenberg Y, Brix K, Schmitz A, Heuser JE, Wilson JH, Lorand L et al. Covalent cross-linking of secreted bovine thyroglobulin by transglutaminase. FASEB Journal $2000141005-$ 1014.

36 Berndorfer U, Wilms H \& Herzog V. Multimerization of thyroglobulin (TG) during extracellular storage: isolation of highly crosslinked TG from human thyroids. Journal of Clinical Endocrinology and Metabolism $1996 \mathbf{8 1} 1918-1926$.
37 Brix K, Lemansky P \& Herzog V. Evidence for extracellularly acting cathepsins mediating thyroid hormone liberation in thyroid epithelial cells. Endocrinology 1996137 1963-1974.

38 Delom F, Mallet B, Carayon P \& Lejeune PJ. Role of extracellular molecular chaperones in the folding of oxidized proteins. Refolding of colloidal thyroglobulin by protein disulfide isomerase and immunoglobulin heavy chain-binding protein. Journal of Biological Chemistry $200127621337-21342$.

39 Delom F, Lejeune PJ, Vinet L, Carayon P \& Mallet B. Involvement of oxidative reactions and extracellular protein chaperones in the rescue of misassembled thyroglobulin in the follicular lumen. Biochemical and Biophysical Research Communications 1999255 438-443.

40 Baudry N, Lejeune PJ, Delom F, Vinet L, Carayon P \& Mallet B. Role of multimerized porcine thyroglobulin in iodine storage. Biochemical and Biophysical Research Communications 1998242 292-296.

41 Schneider PB. Thyroidal iodine heterogeneity: 'last come first served' system of iodine turnover. Endocrinology $1964 \mathbf{7 4}$ 973-980.

Received 12 September 2001

Accepted 7 January 2002 